CERN-TH.7212/94

hep-ph/9404254

\title{
Fragmentation Function Method for Charge Asymmetry Measurements in $e^{+} e^{-}$Collisions
}

\author{
P. Nasont \\ Theory Division, CERN, CH-1211 Geneva 23, Switzerland \\ B.R. Webbert \\ Cavendish Laboratory, University of Cambridge, UK.
}

\begin{abstract}
We propose a method for measuring the hadron charge asymmetry in $e^{+} e^{-}$ collisions which is based upon the fragmentation function formalism, and is largely independent of modelling of fragmentation effects. Furthermore, in this method, QCD radiative corrections can be accounted for in a systematic way.
\end{abstract}

CERN-TH.7212/94

April 1994

* On leave of absence from INFN, Sezione di Milano, Milan, Italy.

${ }^{\dagger}$ Research supported in part by the U.K. Science and Engineering Research Council and by the EC Programme "Human Capital and Mobility", Network "Physics at High Energy Colliders", contract CHRX-CT93-0537 (DG 12 COMA). 


\section{Introduction}

Forward-backward asymmetries measured at $e^{+} e^{-}$colliders near the $Z$ resonance can be used to measure the electroweak parameter $\sin ^{2} \theta_{W}$. The differential cross section for the production of a light fermion pair is given by

$$
\frac{d \sigma_{f}}{d \cos \theta}=\sigma_{f}\left(\frac{3}{8}\left(1+\cos ^{2} \theta\right)+A_{\mathrm{FB}}^{f}(s) \cos \theta\right)
$$

where $\theta$ is the angle of the outgoing fermion $f$ with respect to the direction of the incoming electron. The forward-backward asymmetry is defined as

$$
A_{F B}^{f}=\frac{\sigma_{f}^{F}-\sigma_{f}^{B}}{\sigma_{f}^{F}+\sigma_{f}^{B}}
$$

where $\sigma_{f}^{F}\left(\sigma_{f}^{B}\right)$ is the cross section for producing the fermion in the forward (backward) hemisphere. At leading order on the $Z$ peak (neglecting photon channel contributions) it is given by

$$
A_{F B}^{f}\left(M_{Z}^{2}\right)=\frac{3}{4} \frac{2 v_{e} a_{e}}{v_{e}^{2}+a_{e}^{2}} \frac{2 v_{f} a_{f}}{v_{f}^{2}+a_{f}^{2}}
$$

where

$$
v_{f}=I_{3}^{f}-2 e_{f} \sin ^{2} \theta_{W}, \quad a_{f}=I_{3}^{f},
$$

with $e_{f}$ and $I_{3}$ denoting respectively the charge (measured in units of the positron charge) and the third component of the weak isospin.

Forward-backward asymmetries in the production of leptons are measured at LEP and SLD, and they constitute one very important measurement for electroweak tests (see for example refs. [1]). Due to the fact that $v_{f}$ is very small for leptons, very high precision is required in this channel in order to get a useful measurement of $\sin ^{2} \theta_{W}$. On the other hand, for quarks the vector coupling is not small. There, however, one has difficulty distinguishing between quarks of different flavours, or even between quarks and antiquarks. One approach is that of heavy flavour tagging in the hadronic final state (see refs. [2, 3]). Another possibility is to use instead the total charge asymmetry, averaged over all quark flavours, without flavour tagging. One defines

$$
A_{F B}^{c h}=\frac{1}{\Gamma_{h a d}}\left[\sum_{U=u, c} \Gamma_{U} A_{F B}^{U}-\sum_{D=d, s, b} \Gamma_{D} A_{F B}^{D}\right]
$$


and one then needs to find a final state quantity which is related to the charge of the primary quark, at least on a statistical basis.

Results from various approaches have been reported in refs. [4-6]. The methods used there are all characterized by some "jet charge definition", which should be used to assign a charge to a jet. One then has to establish how often a jet with a given charge comes from a quark with the same charge. This is computed using Monte Carlo simulations. The drawback of this method is that it has to rely upon the hadronization models built into the simulations. A further drawback is that it is not at all clear how the strong corrections to the produced final state affect the measurement. One has no way to understand what part of the radiative correction is already incorporated in the simulation and what part is not. In fact, for a quantity defined in this way, it may be impossible to define the radiative corrections properly.

In the present work, we propose a method for measurement of the charge asymmetry which overcomes some of the above drawbacks: it does not rely so heavily on hadronization models, and QCD radiative corrections are fully and reliably calculable. Our method relies upon the reasonable assumption of valence dominance of the fragmentation functions at large values of the energy fraction $x$, which can be phrased in the following way: at very large $x$, only the hadrons containing a primary quark survive.

In the following sections we give a detailed description of the method, illustrated by some Monte Carlo results obtained using the simulation program HERWIG⿴囗十 [0].

\section{Fragmentation function formalism}

The single inclusive cross section for the production of a charged hadron is given by the formula

$$
\frac{d^{2} \sigma^{h^{ \pm}}}{d x d \cos \theta}=\frac{3}{8}\left(1+\cos ^{2} \theta\right) \frac{d \sigma_{T}^{h^{+}}}{d x}+\frac{3}{4} \sin ^{2} \theta \frac{d \sigma_{L}^{h^{+}}}{d x} \pm \frac{3}{4} \cos \theta \frac{d \sigma_{A}^{h^{+}}}{d x} .
$$

We define the total and asymmetric charged hadron fragmentation functions

$$
F^{h}=\int d \cos \theta\left(\frac{d^{2} \sigma^{h^{+}}}{d x d \cos \theta}+\frac{d^{2} \sigma^{h^{-}}}{d x d \cos \theta}\right)=2\left(\frac{d \sigma_{T}^{h^{+}}}{d x}+\frac{d \sigma_{L}^{h^{+}}}{d x}\right)
$$

\footnotetext{
${ }^{\ddagger}$ We used the current version, HERWIG 5.7, with default parameter values.
} 


$$
F_{A}^{h}=\int d \cos \theta \cos \theta\left(\frac{d^{2} \sigma^{h^{+}}}{d x d \cos \theta}-\frac{d^{2} \sigma^{h^{-}}}{d x d \cos \theta}\right)=\frac{d \sigma_{A}^{h^{+}}}{d x} .
$$

Following the notation of ref. [8] we have

$$
F^{h}(x)=\sum_{f} \sigma_{0, f}(s)\left(D_{f}^{h^{+}}(x, s)+D_{f}^{h^{-}}(x, s)\right)
$$

We use the annihilation scheme, in which the definition of the fragmentation function is such that the coefficient functions for $F^{h}$ have no radiative corrections if the scale in the fragmentation functions is set equal to the centre-of-mass energy squared, $s$. In the following we will drop the dependence on the scale, assuming that it is always set equal to $s$.

In the annihilation scheme, the asymmetry coefficient function does receive higher order corrections. We have

$$
F_{A}^{h}(x)=\sum_{f} A_{f}(s)\left(\bar{D}_{f}^{h^{+}}(x)-\bar{D}_{f}^{h^{-}}(x)\right)
$$

where

$$
\bar{D}_{f}^{h}(x)=D_{f}^{h}(x)-\frac{C_{F} \alpha_{S}}{2 \pi} \int_{x}^{1} \frac{d z}{z}(2-z) D_{f}^{h}(x / z) .
$$

This equation contains all that is needed to compute the radiative corrections of order $\alpha_{S}$. For the moment, we will consider only the leading order analysis, so we will assume that $D_{f}^{h}=\bar{D}_{f}^{h}$.

The electroweak coefficients $\sigma_{0, f}(s)$ and $A_{f}(s)$ are given by the formulae

$$
\begin{aligned}
\sigma_{0, f}(s) & =\frac{4 \pi \alpha^{2}}{s}\left[e_{f}^{2}+2 e_{f} v_{e} v_{f} \rho_{1}(s)+\left(v_{e}^{2}+a_{e}^{2}\right)\left(v_{f}^{2}+a_{f}^{2}\right) \rho_{2}(s)\right] \\
A_{f}(s) & =\frac{8 \pi \alpha^{2}}{s} a_{e} a_{f}\left[e_{f} \rho_{1}(s)+2 v_{e} v_{f} \rho_{2}(s)\right]
\end{aligned}
$$

where

$$
\begin{aligned}
\rho_{1}(s) & =\frac{1}{4 \sin ^{2} \theta_{W} \cos ^{2} \theta_{W}} \cdot \frac{s\left(m_{Z}^{2}-s\right)}{\left(m_{Z}^{2}-s\right)^{2}+m_{Z}^{2} \Gamma_{Z}^{2}} \\
\rho_{2}(s) & =\left(\frac{1}{4 \sin ^{2} \theta_{W} \cos ^{2} \theta_{W}}\right)^{2} \frac{s^{2}}{\left(m_{Z}^{2}-s\right)^{2}+m_{Z}^{2} \Gamma_{Z}^{2}}
\end{aligned}
$$


On the $Z$ peak, neglecting the photon $s$-channel contribution, we have

$$
\begin{aligned}
\sigma_{0, f}(s) & =\frac{4 \pi \alpha^{2}}{s}\left(v_{e}^{2}+a_{e}^{2}\right)\left(v_{f}^{2}+a_{f}^{2}\right) \rho_{2}(s) \\
A_{f}(s) & =\frac{4 \pi \alpha^{2}}{s} 2 a_{e} v_{e} 2 a_{f} v_{f} \rho_{2}(s)
\end{aligned}
$$

\section{Asymmetry from fragmentation functions}

Consider first the total and asymmetric fragmentation functions for pions. According to our valence dominance hypothesis, for large enough $x$ the $\pi^{+}$can only come from a $u$ or a $\bar{d}$ quark. We then have

$$
\begin{aligned}
& F^{\pi}(x)=\sigma_{0, u}(s) D_{u}^{\pi^{+}}(x)+\sigma_{0, d}(s) D_{\bar{d}^{+}}^{\pi^{+}}(x) \\
& F_{A}^{\pi}(x)=A_{u}(s) D_{u}^{\pi^{+}}(x)-A_{d}(s) D_{\bar{d}}^{\pi^{+}}(x)
\end{aligned}
$$

Using isospin symmetry and the on-peak expressions (2.8), we find

$$
\frac{F_{A}^{\pi}(x)}{F^{\pi}(x)}=\left(\frac{2 v_{e} a_{e}}{a_{e}^{2}+v_{e}^{2}}\right) \frac{2 a_{u} v_{u}-2 a_{d} v_{d}}{\left(a_{u}^{2}+v_{u}^{2}\right)+\left(a_{d}^{2}+v_{d}^{2}\right)}=-0.0375
$$

assuming $\sin ^{2} \theta_{W}=0.23$, which we will use as our reference value. The asymmetry is small in the pion case because of the partial cancellation between the $u$ and $d$ contributions.

To test the validity of valence dominance in a particular hadronization model, we studied the behaviour of the pion fragmentation functions using HERWIG. In fig. 1 we plot $F_{A}^{\pi}(x) / F^{\pi}(x)$. The error bars correspond to $2.5 \times 10^{5}$ Monte Carlo events, i.e. about $5 \%$ of the current LEP data. The valence dominance value is also shown. We see that there is agreement for $x>0.5$ at the $10-20 \%$ level. Corrections of this order are not unexpected, for example due to fast non-valence pions from resonance decays. It should be possible to estimate such moderate corrections from hadronization models without introducing a strong model dependence into the determination of $\sin ^{2} \theta_{W}$. Recall that $2 v_{e}=4 \sin ^{2} \theta_{W}-1$, so that a $10 \%$ precision in eq. (3.2) implies about $1 \%$ accuracy in $\sin ^{2} \theta_{W}$.

Let us now consider the production of protons. Again using valence dominance 




Figure 1: Ratio of asymmetry and total fragmentation functions for charged pions, as a function of the energy fraction $x$. The dot-dashed line shows the valence dominance limit of eq. (3.2).

we get

$$
\begin{aligned}
& F^{p}(x)=\sigma_{0, u}(s)\left[D_{u}^{p}(x)+D_{c}^{p}(x)\right]+\sigma_{0, d}(s)\left[D_{d}^{p}(x)+D_{s}^{p}(x)+D_{b}^{p}(x)\right] \\
& F_{A}^{p}(x)=A_{u}(s)\left[D_{u}^{p}(x)+D_{c}^{p}(x)\right]+A_{d}(s)\left[D_{d}^{p}(x)+D_{s}^{p}(x)+D_{b}^{p}(x)\right] .
\end{aligned}
$$

Observe that we have considered as valence dominant the production of protons from strange, charm and bottom quarks. In fact, the production of a strange, charm or bottom baryon will eventually lead through weak decays to a stable nucleon, and in no case (in the valence dominance regime) do we end up with an anti-baryon (baryon) from an initial quark (anti-quark). In the case of charm and bottom baryons, these decays will lead to a considerable degradation of the initial momentum. Since we intend to look at the large- $x$ region, let us neglect for the moment the charm and bottom contributions. We then get

$$
\frac{F_{A}^{p}(x)}{F^{p}(x)}=\left(\frac{2 v_{e} a_{e}}{a_{e}^{2}+v_{e}^{2}}\right) \frac{2 a_{u} v_{u} D_{u}^{p}(x)+2 a_{d} v_{d}\left(D_{d}^{p}(x)+D_{s}^{p}(x)\right)}{\left(a_{u}^{2}+v_{u}^{2}\right) D_{u}^{p}(x)+\left(a_{d}^{2}+v_{d}^{2}\right)\left(D_{d}^{p}(x)+D_{s}^{p}(x)\right)} .
$$

Unlike the case of light mesons, baryon production through resonances does not degrade the baryon momentum substantially. Resonance production and decay tend to 
randomize the charge of the final nucleon. We may therefore, as a first approximation, assume equality of the up, down and strange fragmentation functions to produce protons at large $x$, to obtain

$$
\frac{F_{A}^{p}(x)}{F^{p}(x)}=0.1373
$$

This value is much larger than in the pion case, since no cancellation takes place. In fig. 2 we plot $F_{A}^{p}(x) / F^{p}(x)$. As one can see, the Monte Carlo results approach the value (3.5) very quickly.

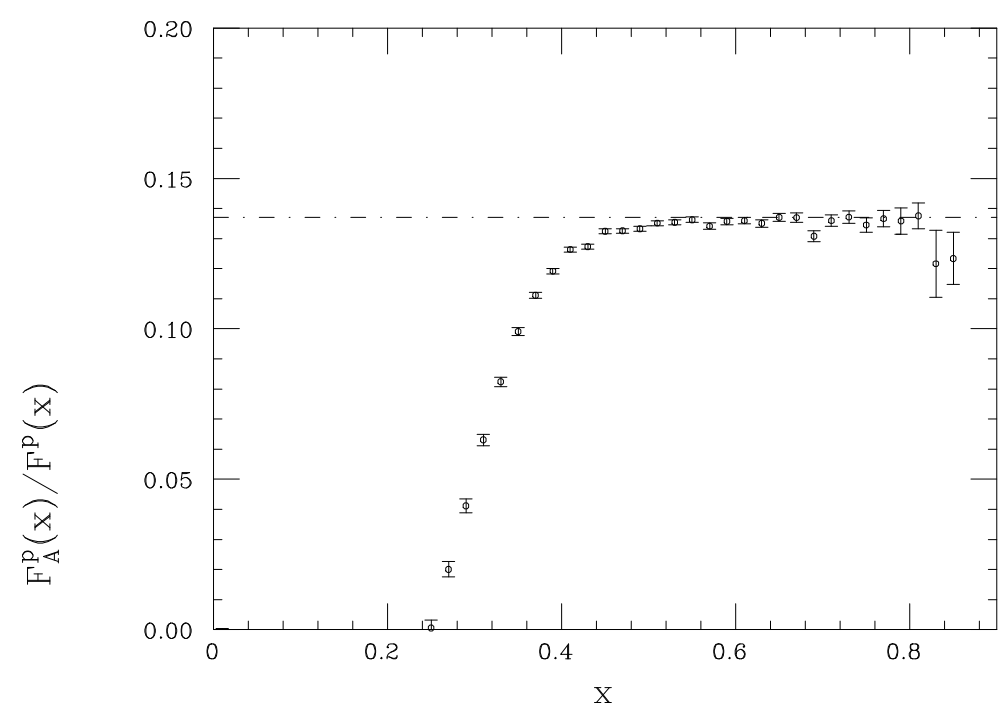

Figure 2: As in fig. 1, but for protons and antiprotons. Dot-dashed: eq. (3.5).

We can also obtain a larger asymmetry in the mesonic case by considering pions and kaons together. We have in the valence dominance approximation (again neglecting the mesons coming from heavy flavour decays)

$$
\frac{F_{A}^{m}(x)}{F^{m}(x)}=\left(\frac{2 v_{e} a_{e}}{a_{e}^{2}+v_{e}^{2}}\right) \frac{2 a_{u} v_{u} D_{u}^{m}(x)-2 a_{d} v_{d}\left(D_{\bar{d}}^{m}(x)+D_{\bar{s}}^{m}(x)\right)}{\left(a_{u}^{2}+v_{u}^{2}\right) D_{u}^{m}(x)+\left(a_{d}^{2}+v_{d}^{2}\right)\left(D_{\bar{d}}^{m}(x)+D_{\bar{s}}^{m}(x)\right)}
$$

where $m$ stands for both charged pions and charged kaons. In this case we would not expect equality of the three fragmentation functions involved. In fact, in the valence dominance limit the only charged meson into which a down quark can fragment is the $\pi^{-}$. By isospin symmetry, $D_{d}^{\pi^{-}}$should equal $D_{u}^{\pi^{+}}$. An up quark, on the other hand, can also go into a $K^{+}$. We therefore expect $D_{u}^{m^{+}}>D_{d}^{m^{-}}$. Our Monte Carlo studies suggest, however, that for $x>0.5$ this difference is less than $20 \%$. 
In the fragmentation of strange quarks, one does not expect much leading pion production. There are however pions coming from neutral kaon decays, which enhance the charged strange fragmentation function without contributing to the charge asymmetry, as assumed in formula (3.6). These effects merit further study, both experimentally and theoretically. For now we will make the rough assumption that the three fragmentation functions are all equal to a first approximation. Under this assumption we obtain

$$
\frac{F_{A}^{m}(x)}{F^{m}(x)} \approx\left(\frac{2 v_{e} a_{e}}{a_{e}^{2}+v_{e}^{2}}\right) \frac{2 a_{u} v_{u}-4 a_{d} v_{d}}{\left(a_{u}^{2}+v_{u}^{2}\right)+2\left(a_{d}^{2}+v_{d}^{2}\right)}=-0.078
$$

i.e. about twice the value in the case of pions alone. Fig. 3 shows a comparison with Monte Carlo results.

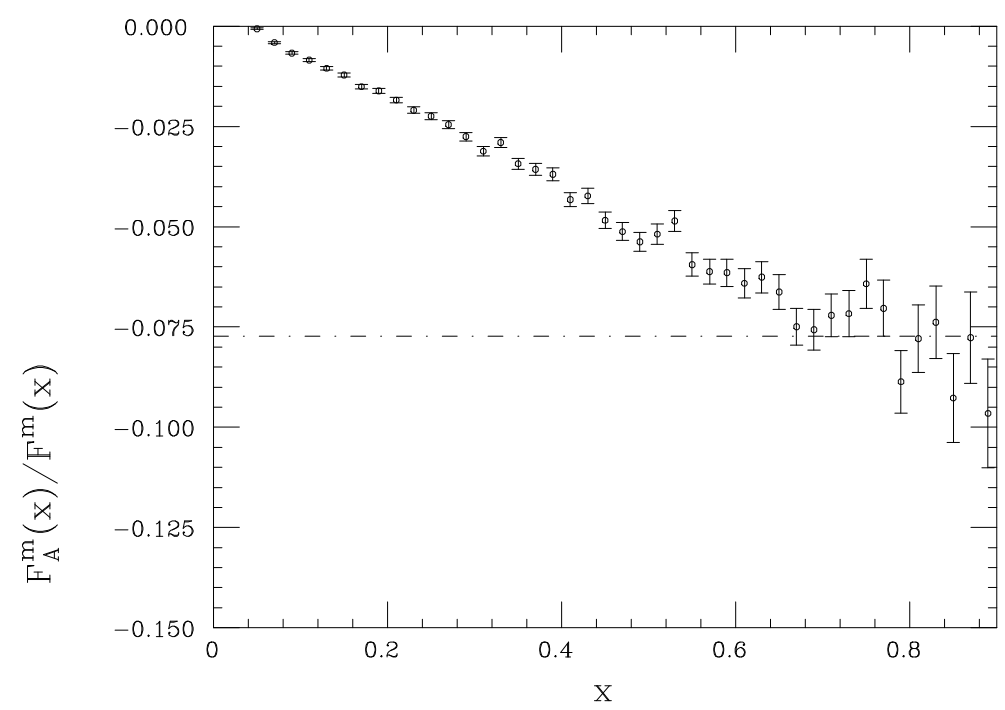

Figure 3: As in fig. 1, but for charged pions plus kaons. Dot-dashed: eq. (3.7).

For completeness, in Fig. Twe also show the total and asymmetric charge meson fragmentation functions $F^{m}(x)$ and $F_{A}^{m}(x)$. The values for $F_{A}^{m}$ are divided by the limiting value of eq. (3.7), to show the relation between the two functions at large $x$. The dashed curve shows the result of applying the higher order correction (2.5) to the total fragmentation function. The effect is small compared with the probable magnitude of the corrections to valence dominance. 


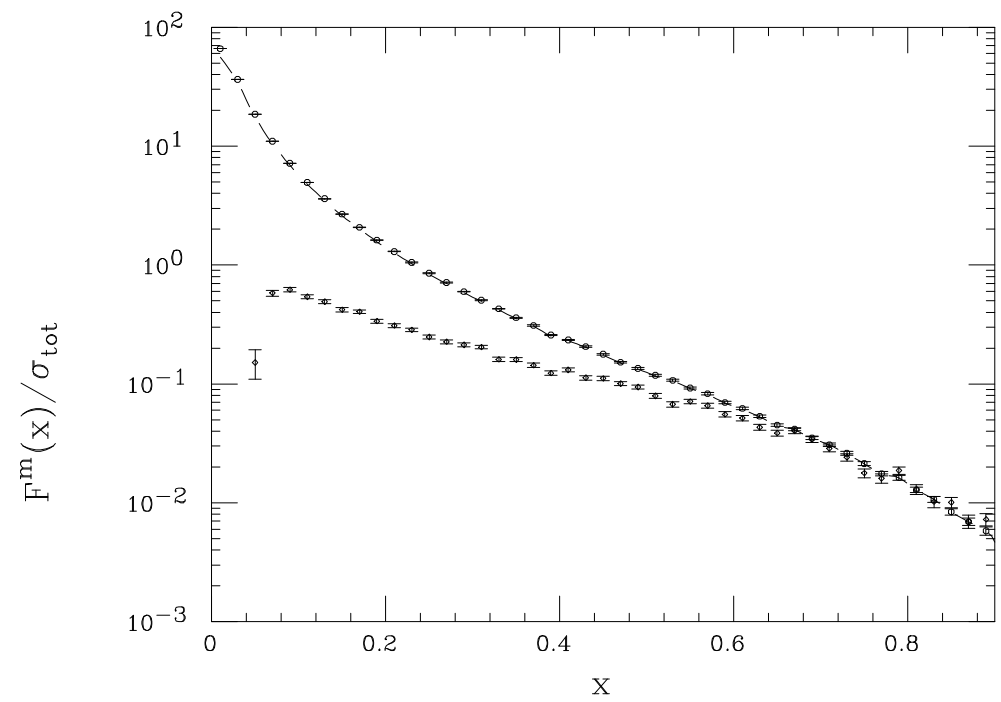

Figure 4: Monte Carlo results on the asymmetry and total fragmentation functions for charged mesons, (pions plus kaons), as functions of the energy fraction $x$, at $E_{c . m} .=91.2 \mathrm{GeV}$. The asymmetry has been divided by its valence dominance limiting value of eq. (3.2). Dashed curve: result of applying the higher order correction (2.5) to the total fragmentation function.

\section{Conclusions}

The method we propose for studying hadronic charge asymmetries, and hence measuring $\sin ^{2} \theta_{W}$, is based directly on the fragmentation function formalism, for which the machinery of perturbative QCD is already well developed. In particular the relation between the total and asymmetric fragmentation functions for individual flavours is known, and measurements at different energies can be related, to next-toleading order in $\alpha_{S}$. This makes the method attractive from the theoretical viewpoint.

For phenomenological applications, one needs to exploit relationships between the fragmentation functions of quarks and their flavours. The type of valence dominance we have assumed seems less model dependent than other relationships between quark and jet properties that have been proposed for this purpose. Typical corrections are at the 10-20\% level at high $x$ and could be safely estimated from models. The predicted asymmetries for protons and light mesons at the $Z$ resonance are substantial and of opposite sign, providing a valuable cross-check on the method.

Other cross-checks may be obtained experimentally. One can, for example, require 
one fast particle in one jet, and then study the fragmentation functions of the opposite jet. For example, if one requires a fast $\pi^{+}$on one side, (which, according to valence dominance, can only come from a $u$ or a $\bar{d}$ quark), this implies that on the opposite side we can measure the linear combination of fragmentation functions $\left(v_{u}^{2}+a_{u}^{2}\right) D_{\bar{u}}^{h}+$ $\left(v_{d}^{2}+a_{d}^{2}\right) D_{d}^{h}$ directly, for any $x$ value. This in turn can be used to test the valence dominance hypothesis itself, and to measure the $K$ and $p$ fragmentation functions for this combination. This measurement can in turn be used to correct for deviations from the valence dominance hypothesis. Many other combinations (for example requiring a fast proton or $\Lambda_{s}$ or a kaon on one side) can be studied, allowing for a wide variety of consistency checks and error estimates which are independent of hadronization models.

We have concentrated here on light-quark hadrons, but of course the method can also be applied to heavy quark fragmentation. In particular the asymmetric and total $D^{*}$ fragmentation functions at large $x$ would provide a rather clean measurement based on charmed quarks.

\section{Acknowledgements}

We acknowledge useful discussions with P. Antilogus, A. Blondel, D. Rees, P. Perrodo and R. Jones.

\section{References}

1. ALEPH Collab., D. Buskulic et al., preprints CERN-PPE/93-40, CERN-PPE/9430 ;

DELPHI Collab., P. Abreu et al., Z. Phys. C59(1993)21; preprint CERNPPE/94-31;

L3 Collab., B. Adeva et al., Z. Phys. C51(1991)179; preprint CERN-PPE/9368 ;

OPAL Collab., R. Akers et al., Z. Phys. C61(1994)19.

2. L3 Collab., O. Adriani et al., Phys. Lett. B292(1992)454; preprint CERNPPE/93-68;

OPAL Collab., P.D. Acton et al., Z. Phys. C60(1993)601;

TOPAZ Collab., E. Nakano et al., Phys. Lett. B314(1993)471. 
3. A. Djouadi, J.H. Kühn and P.M. Zerwas, Z. Phys. C46(1990)441;

G. Altarelli and B. Lampe, Nucl. Phys. B391(1993)3;

B. Lampe, preprint MPI-Ph/93-74.

4. ALEPH Collab., D. Decamp et al., Phys. Lett. B259(1991)377.

5. DELPHI Collab., P. Abreu et al., Phys. Lett. B277(1992)371.

6. OPAL Collab., P.D. Acton et al., Phys. Lett. B294(1992)436.

7. G. Marchesini and B.R. Webber, Nucl. Phys. B310(1988)461;

G. Abbiendi, I.G. Knowles, G. Marchesini, B.R. Webber, M.H. Seymour and L. Stanco, Computer Phys. Comm. 67(1992)465.

8. P. Nason and B.R. Webber, preprint CERN-TH.7018/93, to be published in Nucl. Phys. B. 